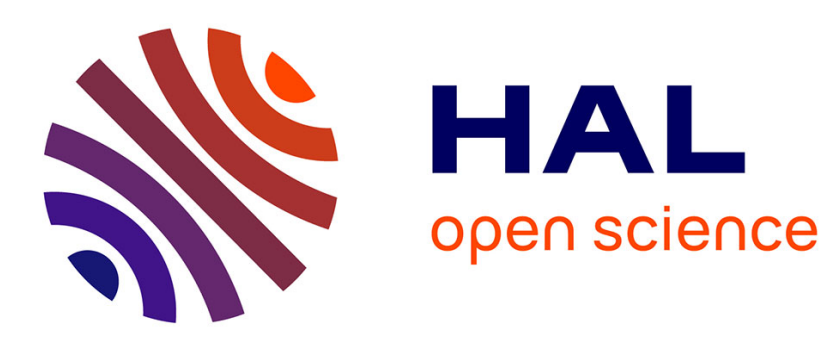

\title{
CORROSION INTERGRANULAIRE.SUR LE MÉCANISME DE LA CORROSION INTERGRANULAIRE DES MATÉRIAUX MÉTALLIQUES
}

Michel Froment

\section{To cite this version:}

Michel Froment. CORROSION INTERGRANULAIRE.SUR LE MÉCANISME DE LA CORROSION INTERGRANULAIRE DES MATÉRIAUX MÉTALLIQUES. Journal de Physique Colloques, 1975, 36 (C4), pp.C4-371-C4-385. 10.1051/jphyscol:1975438 . jpa-00216344

HAL Id: jpa-00216344

https://hal.science/jpa-00216344

Submitted on 1 Jan 1975

HAL is a multi-disciplinary open access archive for the deposit and dissemination of scientific research documents, whether they are published or not. The documents may come from teaching and research institutions in France or abroad, or from public or private research centers.
L'archive ouverte pluridisciplinaire HAL, est destinée au dépôt et à la diffusion de documents scientifiques de niveau recherche, publiés ou non, émanant des établissements d'enseignement et de recherche français ou étrangers, des laboratoires publics ou privés. 


\title{
SUR LE MÉCANISME DE LA CORROSION INTERGRANULAIRE DES MATÉRIAUX MÉTALLIQUES
}

\author{
M. FROMENT
}

Physique des Liquides et Electrochimie

Groupe de Recherche $\mathrm{n}^{\circ} 4$ du C.N.R.S.

Associé à l'Université Pierre-et-Marie-Curie

4, place Jussieu, 75230 Paris Cedex 05, France.

\begin{abstract}
Résumé. - L'article est consacré à l'étude des phénomènes de corrosion intergranulaire présentés par les métaux et alliages exempts de toute précipitation intergranulaire. On s'efforce de relier l'attaque intergranulaire à un certain nombre de données importantes concernant les joints de grains : énergie, structure cristallographique, ségrégation d’impuretés... On étudie l'influence de l'énergie et de la structure cristallographique à l'aide de séries de bicristaux d'aluminium et d'aciers inoxydables présentant des joints de flexion d'axes $\langle 100\rangle$ et $\langle 110\rangle$. La microscopie électronique à balayage permet d'obtenir des données quantitatives sur l'attaque intergranulaire. On présente également des résultats concernant les phénomènes de ségrégation dans le cas d'échantillons polycristallins de nickel et d'aciers au chrome-nickel ayant diverses teneurs en carbone et en silicium. On propose un mécanisme d'attaque intergranulaire tenant compte des courants de dissolution de la matrice et des joints de grains. Ce mécanisme explique la formation de sillons d'attaque présentant des caractéristiques géométriques bien définies.
\end{abstract}

\begin{abstract}
This paper is devoted to the study of intergranular corrosion phenomena of metals and alloys observed even when these materials are free from any intergranular precipitate. We endeavour to correlate the specific features of this intergranular attack with several important data pertaining to grain boundaries : energy, crystallographic structure, impurities segregation...

The influence of energy and crystallographic structure is studied using series of aluminium and stainless steels bicrystals with tilt boundaries along $\langle 100\rangle$ and $\langle 100\rangle$ axis. Scanning electron microscopy permits to obtain quantitative data on the intergranular attack. Some results are given on segregation phenomena in the case of polycrystalline nickel and chromium-nickel steels with variables contents of carbon and silicon. We propose a tentative mechanism for intergranular corrosion involving the dissolution currents of both the matrix and the grain boundaries. This mechanism explains the formation of etching grooves of well defined geometrical features.
\end{abstract}

Introduction. - La corrosion intergranulaire correspond à une dissolution préférentielle des zones d'émergence des joints de grains à la surface des matériaux mis en présence de milieux agressifs. Les milieux susceptibles d'entraîner l'attaque intergranulaire sont très variés: gaz, solutions, sels fondus, métaux liquides... Les phénomènes observés ont plusieurs origines. Ainsi, la précipitation de phases étrangères au voisinage des joints de grains peut entraîner, suivant la nature de l'environnement agressif, la dissolution préférentielle des précipités ou des zones voisines des précipités appauvries en un élément constituant la matrice. C'est le cas de la corrosion intergranulaire de certains alliages légers et des aciers inoxydables, austénitiques ou ferritiques, sensibilisés. Nous ne traiterons pas ici de ce type de corrosion qui a fait l'objet de nombreuses mises au point ces dernières années, soit pour les alliages légers [1], soit pour les aciers à base de nickel et de chrome [2, 3]. De la même façon, nous ne discuterons pas des phénomènes de corrosion sous contrainte ou de fragilisation par l'hydrogène qui entraînent une attaque ou une décohésion ntergranulaire. Une analyse approfondie de ces phénomènes a été faite au cours de 2 Colloques internationaux en 1972 et $1973[4,5]$. Nous nous limiterons donc aux phénomènes d'attaque intergranulaire en phase homogène. Ces phénomènes sont connus et étudiés depuis fort longtemps dans le cas de l'aluminium [6,7]. Ils ont donné lieu, ces dernières années, à d'assez nombreux travaux portant sur les alliages à base de fer, nickel et chrome. En effet, ces matériaux sont mis en œuvre, en particulier dans le génie chimique ou nucléaire, dans des conditions de plus en plus difficiles (températures élevées, milieux très oxydants...) et ils se sont alors révélés sensibles à ce type de corrosion $[8,9,10,11]$.

Sur le plan fondamental, l'étude de la corrosion intergranulaire en phase homogène présente un intérêt certain. En effet, elle conduit à l'établissement de corrélations entre les caractéristiques 
morphologiques de l'attaque et des propriétés intrinsèques des joints de grains. De tels travaux peuvent donc apporter une contribution à la connaissance des joints de grains.

1. Méthodes d'étude de la corrosion intergranulaire. - La plupart des travaux ont été effectués avec des matériaux polycristallins. Cependant, la mise en cuvre, depuis un certain nombre d'années, de bicristaux présentant des joints aux caractéristiques bien déterminées, a conduit à une meilleure connaissance du comportement des joints de grains vis-à-vis de la corrosion. Les bicristaux ont surtout été utilisés pour l'étude de la corrosion intergranulaire des aciers inoxydables $[12,13,14]$, et de l'aluminium $[15,16,17]$. On peut espérer que les progrès réalisés par les techniques d'élaboration permettront d'aborder les études de corrosion intergranulaire de la plupart des matériaux métalliques sous forme de bicristaux. Un autre point à souligner concerne la mise en œuvre de matériaux très purs. Des travaux effectués ces dernières années ont révélé le rôle important joué par certaines impuretés dans la corrosion intergranu. laire de l'aluminium [18, 19] et des aciers inoxydables [20].

Les études sur la corrosion intergranulaire ont largement bénéficié des progrès effectués par les techniques potentiostatiques, potentiocinétiques... d'alimentation des cellules d'électrolyse [21]. Il en est résulté une bien meilleure définition des conditions électrochimiques de l'attaque (contrôle et stabilité de la tension d'électrode, du courant, de la quantité d'électricité ayant traversé la cellule...) que lors de la mise en œuvre de tests de corrosion basés sur l'immersion dans des milieux acides (tests de Huey, de Strauss...).

Enfin, les données morphologiques sur l'attaque intergranulaire ont pu être dégagées avec beaucoup plus de précision depuis que les techniques de microscopie électronique ont été largement mises à contribution. On soulignera l'intérêt de la microscopie électronique à balayage dont la profondeur de champ très importante est bien adaptée à l'étude des phénomènes de corrosion localisée $[22,23]$. La microscopie électronique par transmission garde un intérêt certain pour l'étude des premiers stades de la corrosion [24]. La préparation de lames minces subissant ultérieurement un traitement d'attaque de faible importance permet d'observer l'initiation des phénomènes et l'établissement de corrélations entre les sites où démarre l'attaque et la nature des défauts structuraux [23]. Cette technique nécessite l'utilisation de lames relativement épaisses et leur observation en microscopie électronique à haute tension est particulièrement intéressante $[25,26]$.

2. Initiation de la corrosion intergranulaire. Différents arguments ont été avancés pour expli- quer le démarrage d'une attaque préférentielle des zones d'émergence des joints de grains.

Sans faire d'hypothèses sur la structure des joints de grains, on peut admettre que l'existence de zones à énergie élevée modifie localement les conditions thermodynamiques de stabilité du matériau [27]. Si l'on considère un cycle amenant les atomes du réseau métallique à l'état d'ions solvatés [28], on voit immédiatement que les atomes situés au voisinage des joints de grains, ayant une énergie plus importante, passent plus facilement en solution. L'énergie $\Delta G$ correspondant aux zones intergranulaires entraîne une variation $\Delta V \mathrm{du}$ potentiel d'équilibre :

$$
\Delta V=-\frac{\Delta G}{Z F}
$$

où $Z$ est la valence des ions passant en solution et $F$ le Faraday. En prenant par exemple le fer $\gamma$ (énergie moyenne des joints de grande désorientation voisine de $850 \mathrm{ergs} / \mathrm{cm}^{2}$ ), et en admettant que la largeur du joint correspond à 3 fois le paramètre $a$ du réseau $(a=3,6 \AA)$, on obtient $\Delta G=1,68 \mathrm{Kcal} \mathrm{mole}^{-1}$. La différence de potentiel qui en résulte serait alors de $36,4 \mathrm{mV}$ valeur non négligeable.

Un autre point de vue, probablement plus réaliste, est de considérer la cinétique de dissolution anodique des zones intergranulaires [27]. En effet, un milieu agressif donné impose un potentiel de corrosion constant lié aux réactions d'oxydoréduction intervenant à la surface de l'électrode. Par ailleurs, comme nous le verrons plus loin, l'existence de vitesses de dissolution différentes de la matrice et du joint de grains expliquerait la formation de sillons d'attaque ayant un profil stable. Le courant de dissolution anodique Ia s'exprime par la relation:

$$
I \mathrm{a}=I_{0} \mathrm{e}^{b V}
$$

A potentiel constant $V$, la présence de zones intergranulaires pourrait modifier le terme préexponentiel $I_{0}$ :

$$
I_{0}=K \mathrm{e}^{-E / R T}
$$

où $K$ est proportionnel au nombre de sites superficiels actifs et $E$ l'énergie d'activation. On peut alors prévoir une augmentation du nombre de sites actifs liée à l'émergence de défauts de réseau et/ou une variation de l'énergie d'activation $E$ liée à l'énergie des zones intergranulaires. Si l'on suppose que la variation de $E$ est du même ordre de grandeur que $\Delta G\left(1,68 \mathrm{Kcal} \mathrm{mole}^{-1}\right)$, il en résulterait un courant de dissolution des zones intergranulaires au moins 10 fois supérieur à celui de la matrice. Des expériences effectuées sur des aciers inoxydables contenant du silicium ont effectivement révélé des variations entre les courants de dissolution anodique des joints et de la matrice de cet ordre de 
grandeur [29]. Il est cependant difficile dans ce cas de séparer les facteurs liés à la variation d'énergie intergranulaire et ceux dus à une ségrégation intergranulaire du silicium.

On peut également considérer que le joint émerge sous forme de défauts cristallins (dislocations), fournissant ainsi des sites pour l'initiation de l'attaque. On sait en effet que sur une surface cristallographiquement parfaite le démarrage de l'électrocristallisation ou de la dissolution anodique nécessite une surtension minimale; lorsqu'on dépasse cette surtension, il y a formation de germes de croissance [30] ou de décroissance [31]. D'après les travaux de Kaischew et Budevski [30], une surtension minimale de l'ordre de $10 \mathrm{mV}$ est nécessaire pour la formation de germes bidimensionnels sur une surface d'argent monocristalline parfaite. Or, lorsque des dislocations émergent à la surface, la surtension minimale provoquant l'électrocristallisation ou la dissolution anodique est très nettement diminuée. Des expériences de Nanev et Kaischew [32] ont prouvé, par envoi dans la cellule d'impulsions de tension cathodique, provoquant la formation de pyramides à l'émergence des dislocations, puis d'impulsions anodiques, que le démarrage de l'attaque s'effectuait bien aux mêmes sites d'émergence des dislocations. Des maintiens potentiostatiques effectués sur des surfaces monocristallines d'argent ont par ailleurs conduit à préciser les surtensions minimales pour l'attaque de l'argent en présence de dislocations [33]: $1 \mathrm{mV}$ sur le plan $\{100\}$ et $2,5 \mathrm{mV}$ sur le plan $\{111\}$. Le modèle de formation de germes de décroissance à l'émergence des dislocations conduit à un bon accord avec l'expérience à condition de prendre des énergies d'incorporation d'un atome d'argent sur un gradin de, respectivement: $1,2 \times 10^{-6}$ et $1,8 \times 10^{-6} \mathrm{erg} \mathrm{cm}^{-1}$ pour les plans $\{100\}$ et $\{111\}$. Ces valeurs sont très voisines de celles déduites par Budevski au cours d'expériences d'électrocristallisation de l'argent. Le modèle proposé par Engell [33] conduit également à prévoir des germes de décroissance dont le rayon critique serait inférieur à $100 \AA$. L'existence d'une surtension d'attaque plus faible des défauts cristallographiques expliquerait donc le démarrage de l'attaque au niveau des joints de grains.

Toutes les considérations qui viennent d'être développées impliquent qu'au cours de l'attaque, le matériau métallique est en contact direct avec le milieu agressif. En fait, l'attaque intergranulaire intervient fréquemment dans des conditions où une couche plus ou moins épaisse et protectrice recouvre la surface. C'est en particulier le cas de l'aluminium où une couche d'oxyde formée à l'air persiste même après des maintiens prolongés dans des solutions aqueuses d'acide chlorhydrique. Les maintiens en corrosion libre dans ce milieu provoquent une attaque intergranulaire et la couche d'oxyde n'est détruite par corrosion localisée qu'après des maintiens à une surtension anodique d'au moins $0,2 \mathrm{~V}$. L'attaque intergranulaire du nickel [34] ou des aciers inoxydables [35] dans des solutions aqueuses d'acide sulfurique est par ailleurs très importante au début du domaine de transpassivité $(V \sim 1,1 \mathrm{~V} / \mathrm{E}$.C.S. $)$; dans ces conditions, la couche passive n'est pas complètement détruite. On peut donc admettre une perturbation de la couche à l'aplomb des joints de grains, perturbation qui entraînerait une dissolution localisée. Le phénomène d'attaque intergranulaire serait alors d'autant plus sévère que la surface est recouverte d'une couche limitant la vitesse de dissolution. Il est certain également que la présence d'impuretés ségrégées au joint peut amorcer l'attaque intergranulaire. L'importance de ces impuretés est peut-être encore plus décisive lorsque la surface est recouverte d'une couche protectrice. En effet, ces impuretés seraient susceptibles de se concentrer dans la couche. Or, on sait qu'en présence de certains éléments d'addition, les couches passives voient leurs propriétés de conduction complètement modifiées [36]. D'ailleurs, les modifications des propriétés des couches passives, par des impuretés, ont souvent été invoquées pour expliquer l'initiation du phénomène de corrosion localisée $[37,38]$. Nous ne discuterons pas ici plus en détail des phénomènes d'initiation de la corrosion intergranulaire, en relation avec la présence de couches protectrices, car ils font l'objet d'une communication au cours de la même session [76].

3. Propagation de la corrosion intergranulaire ; évaluation de son importance. - Dans la plupart des cas, on observe, après une période transitoire, que la corrosion intergranulaire atteint un régime stable: à l'aplomb de tous les joints de grains apparaissent des sillons plus ou moins profonds. La figure 1 montre l'aspect d'un échantillon de nickel polycristallin contenant $1 \%$ de silicium après un maintien à $1,1 \mathrm{~V} / \mathrm{E} . \mathrm{C} . \mathrm{S}$. dans une solution $2 \mathrm{~N}$

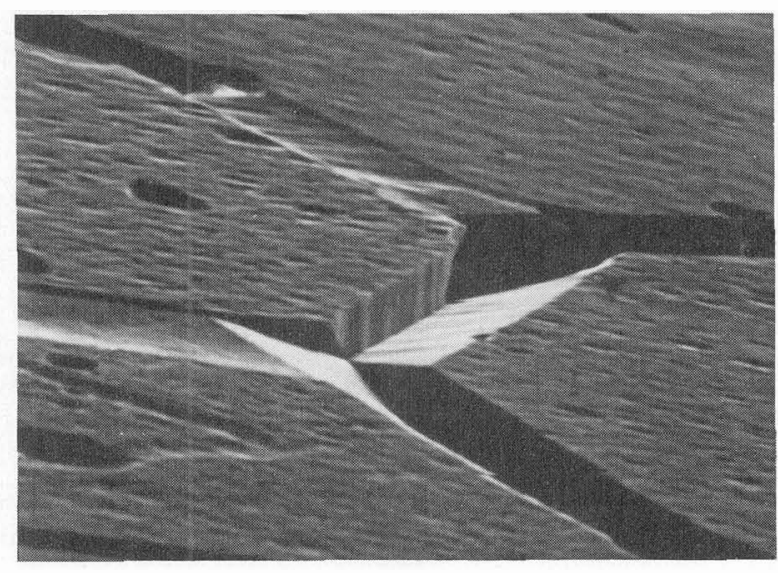

FIG, 1. - Examen en microscopie électronique à balayage de l'attaque intergranulaire de nickel polycristallin contenant $1 \%$ de silicium; $\mathrm{H}_{2} \mathrm{SO}_{4} 2 \mathrm{~N}, V=1,1$ V/E.C.S. $G=850$. 
d'acide sulfurique; on constate que les joints sont plus ou moins attaqués. Les sillons d'attaque sont beaucoup plus réguliers dans le cas de bicristaux comme on peut le voir sur la figure 2 relative également à du nickel. La figure 3 correspond à un bicristal d'aluminium attaqué dans une solution aqueuse d'acide chlorhydrique ; cette image comme celle de la figure 2 a été obtenue en inclinant très fortement $\left(82^{\circ}\right)$ l'objet par rapport au faisceau. On peut alors apprécier l'importance de l'attaque intergranulaire par les caractéristiques géométriques du sillon formé à l'aplomb du joint : la largeur $L$, la profondeur $H$, et l'angle d'ouverture $\alpha$ sont mesurés directement sur le cliché. Le phénomène d'attaque intergranulaire est d'autant plus sévère qu'après une exposition à un milieu agressif dans des conditions données, l'angle d'ouverture $\alpha$ est plus petit et les valeurs de $L, H$ plus importantes.

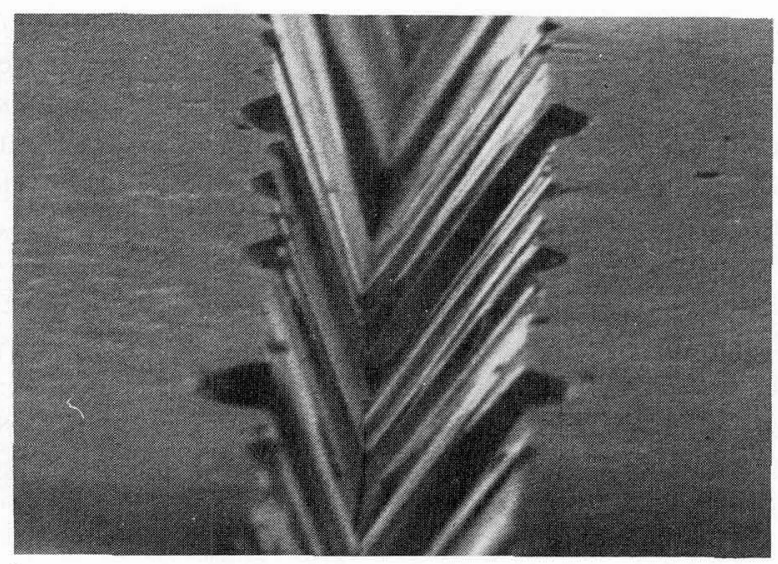

FIG. 2. - Aspect du sillon d'attaque d'un bicristal de nickel d'axe de flexion $\langle 100\rangle$ et présentant une désorientation de $43^{\circ} ; \mathrm{H}_{2} \mathrm{SO}_{4} 2 \mathrm{~N}, V=1,3$ V/E.C.S. $G=2100$.

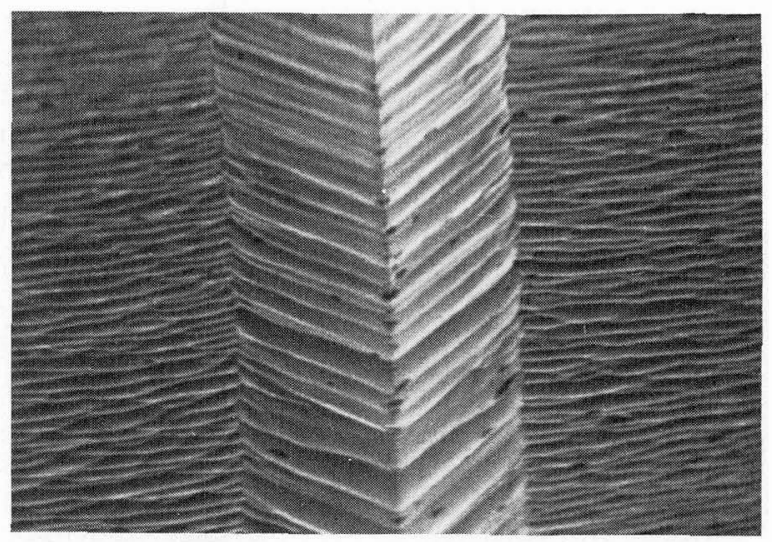

FIG. 3. - Attaque intergranulaire d'un bicristal d'aluminium d'axe de flexion $\langle 100\rangle$ et présentant une désorientation de $40^{\circ}$; maintien de 290 heures dans $\mathrm{HCl} 10 \% . G=7600$.

Si l'on étudie l'évolution des caractéristiques géométriques du sillon d'attaque en fonction du temps, on constate généralement qu'après une variation rapide, l'angle d'ouverture $\alpha$ atteint une valeur stable [39]. Celle-ci est obtenue après le passage d'une quantité d'électricité qui dépend du matériau et des conditions de l'attaque. On se placera donc dans des conditions conduisant à un régime stable, c'est-à-dire où le sillon a atteint un profil d'équilibre. Ainsi, sera facilitée l'étude des différents facteurs intervenant sur la corrosion intergranulaire. On voit tout de suite que dans le cas d'échantillons polycristallins, on sera amené à caractériser la corrosion intergranulaire par des valeurs moyennes relatives à un nombre important de joints à forte désorientation. Par contre, la mise en cuvre de bicristaux aux caractéristiques cristallographiques connues permettra de rattacher beaucoup plus facilement l'importance de la corrosion intergranulaire à des données comme la désorientation entre les grains, l'asymétrie des joints... Enfin, l'utilisation des bicristaux permet de tenir compte des facteurs d'anisotropie de l'attaque suivant que celle-ci est effectuée parallèlement ou perpendiculairement à l'axe, dans le cas des joints de flexion.

4. Les facteurs de la corrosion intergranulaire. 4.1 ENERGIE DES JOINTS DE GRAINS. - L'existence d'une relation entre l'importance de l'attaque intergranulaire et l'énergie des joints a été signalée depuis longtemps. Celle-ci est particulièrement nette aux faibles valeurs de la désorientation $\theta\left(0<\theta<20^{\circ}\right)$. Ainsi, Leggett et Paxton [12] ont trouvé une augmentation de l'attaque intergranulaire avec la désorientation, dans le cas d'échantillons d'acier inoxydable formés de gros grains immergés dans l'acide nitrique bouillant. Des résultats analogues ont été signalés par Mieluch et Smialowski au cours de la polarisation anodique du fer dans une solution de nitrate d'ammonium [40]. Les travaux systématiques effectués ces dernières années avec des bicristaux ont confirmé cette relation. Ainsi, dans le cas des bicristaux d'aluminium possédant des joints de flexion symétriques d'axe $<100>$ ou $<110>$, on trouve une augmentation plus ou moins rapide de la corrosion intergranulaire, soit dans l'eau pressurisée [41], soit dans une solution aqueuse d'acide chlorhydrique [42], lorsque la désorientation s'élève jusqu'à une vingtaine de degrés. Des observations analogues ont été faites dans le cas de l'attaque intergranulaire de bicristaux d'acier inoxydable d'axe de flexion $<100>$ maintenus au début du domaine de transpassivité dans une solution aqueuse d'acide sulfurique [43]. Comme nous le verrons ultérieurement, l'augmentation plus ou moins rapide de l'attaque intergranulaire suivant que l'on se trouve au voisinage de 0 ou $90^{\circ}$ de désorientation peut s'expliquer par une différence dans les indices des plans cristallins formant la surface du bicristal au contact avec l'électrolyte ou par la nature des défauts cristallins constituant les joints de grains.

L'influence de l'énergie des joints sur la corro- 
sion intergranulaire est beaucoup plus difficile à dégager lorsqu'on atteint le domaine des fortes désorientations. Dans le cas des échantillons polycristallins d'acier inoxydable ou de nickel contenant de 0 à $5 \%$ de silicium, on a trouvé que la corrosion intergranulaire était d'autant plus importante que l'énergie des joints était plus élevée [44, 45]; l'énergie des joints a été évaluée par mesure des profils d'équilibre des sillons intergranulaires après attaque thermique, ou par application de la formule de Borisov à l'autodiffusion intergranulaire d'un traceur radioactif $[46,47]$. Cependant, il est très difficile, dans le cas de ces matériaux, de séparer les facteurs jouant directement sur l'énergie intergranulaire de ceux modifiant la composition de la matrice ou entraînant des ségrégations intergranulaires. Ces deux derniers facteurs conduisent, comme nous allons le voir, à des variations importantes des vitesses de dissolution de la matrice et des zones intergranulaires. Il est donc plus facile de tenter une analyse des relations entre énergie et corrosion intergranulaire dans le cas de matériaux ayant la même composition et se présentant sous forme de bicristaux. Un certain nombre de résultats semblent confirmer que la corrosion intergranulaire est d'autant plus importante que l'énergie des joints est plus élevée. La figure 4 tirée de la thèse de J. Y. Boos [41] montre l'évolution de

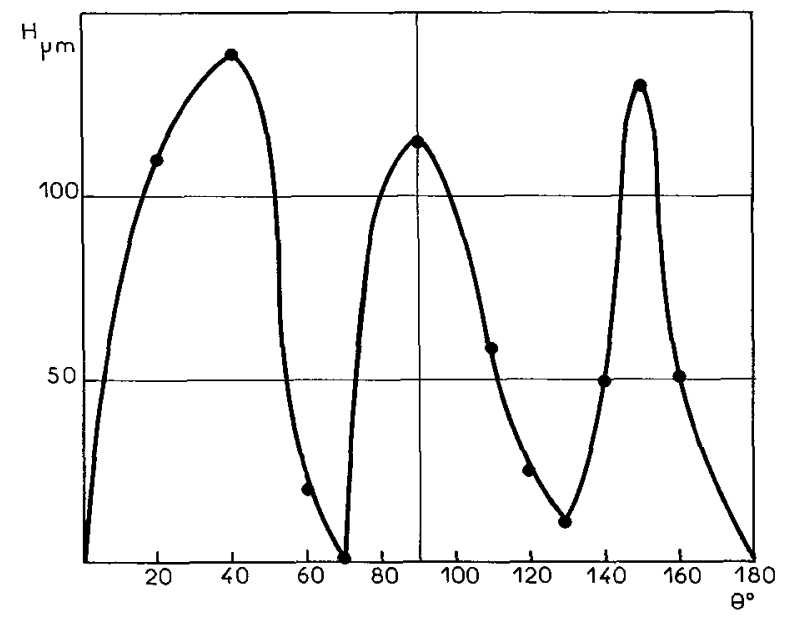

FIG. 4. - Influence de la désorientation $\theta$ sur la profondeur d'attaque $H$ de bicristaux d'axe de flexion $\langle 110\rangle$ après maintien dans l'eau pressurisée à $150^{\circ} \mathrm{C}$ (Thèse J. Y. Boos, 1971).

la profondeur $H$ du sillon d'attaque mesurée sur des bicristaux d'aluminium mis en présence d'eau pressurisée ; les joints symétriques, d'axe de flexion $\langle 011\rangle$ ont été attaqués perpendiculairement à l'axe de flexion. Cette courbe est en étroite corrélation avec l'évolution de l'énergie intergranulaire calculée suivant une méthode proposée par Hasson [16, 48]. En particulier, les macles $\{111\}-70^{\circ} 30^{\prime},\{311\}-129^{\circ} 30^{\prime}$, sont très peu attaquées.
La corrosion intergranulaire de bicristaux d'aciers inoxydables ( $\mathrm{Cr} 17 \%, \mathrm{Ni} 13,5 \%$ ), effectuée dans une direction perpendiculaire à l'axe de flexion $\langle 100\rangle$ conduit également à une assez bonne relation entre corrosion et énergie intergranulaire [43]. La figure 5 montre l'évolution de $L$ et $2 \cos \alpha / 2$ en fonction de la désorientation $\theta$. L'attaque a été effectuée dans une solution d'acide sulfurique $2 \mathrm{~N}$ maintenue à $50^{\circ} \mathrm{C}$, la tension étant fixée à 1,1 V/E.C.S. La courbé donnant l'évolution de $2 \cos \alpha / 2$ suit assez bien l'énergie intergranulaire calculée dans le cas d'un métal cubique à faces c entrées; en particulier, les macles $\{013\}-\theta=37^{\circ}, \quad\{012\}-\theta=53^{\circ}$, $\{023\}-\theta=67^{\circ}$ se distinguent nettement.

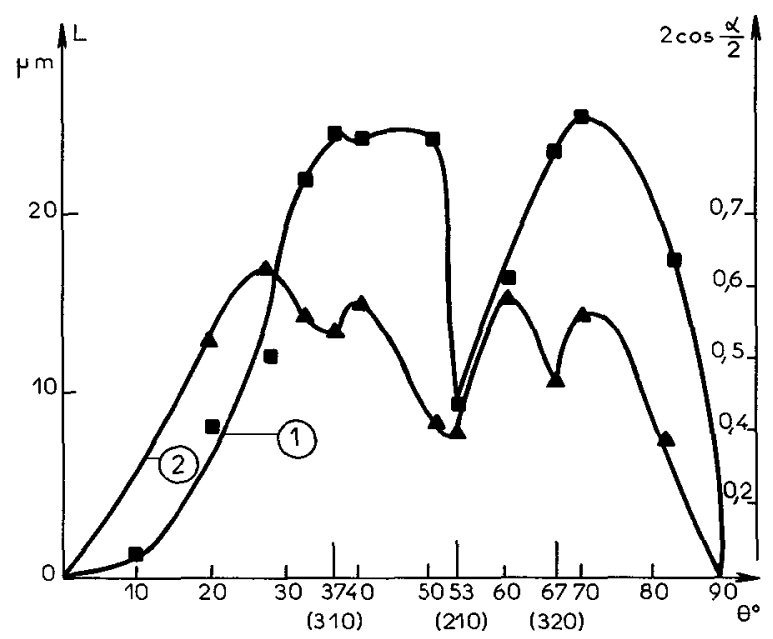

FIG. 5. - Influence de la désorientation $\theta$ sur la largeur du sillon d'attaque $L$ (courbe 1 ) et le paramètre $2 \cos \alpha / 2$ (courbe 2) après attaque perpendiculaire à l'axe de flexion $\langle 100\rangle$ de bicristaux d'acier inoxydable dans $\mathrm{H}_{2} \mathrm{SO}_{4} 2 \mathrm{~N}$ à $1,1 \mathrm{~V} / \mathrm{E}$.C.S.

Cependant, dans de nombreux cas, il n'est pas possible de rattacher aussi étroitement corrosion et énergie intergranulaires. Il est difficile également d'expliquer la très grande anisotropie présentée par la corrosion intergranulaire. Ainsi, la figure 6 montre l'évolution de la hauteur $H$ des sillons après un maintien de 290 heures dans l'acide chlorhydrique à $10 \%$, de bicristaux d'aluminium; ces derniers, d'axe de flexion $\langle 100\rangle$ ont été attaqués dans une direction soit parallèle, soit perpendiculaire à l'axe de flexion; l'importance de l'attaque en dépend beaucoup [17]. Par ailleurs, la macle $\{310\}-\theta=37^{\circ}$ ne se distingue pas des autres joints, surtout lorsque l'attaque est effectuée parallèlement à l'axe. Des conclusions analogues peuvent être déduites de la figure 7 relative à des bicristaux d'aluminium d'axe de flexion $\langle 110\rangle$. Dans ce cas, les maximums d'attaques pour des désorientations de $40-60^{\circ}$ et $150-160^{\circ}$ correspondent assez bien aux maximums d'énergie intergranulaire [48]. Cependant, il n'est pas possible d'expliquer le très faible niveau de l'attaque au voisinage de $100^{\circ}$ où l'énergie intergranulaire est 


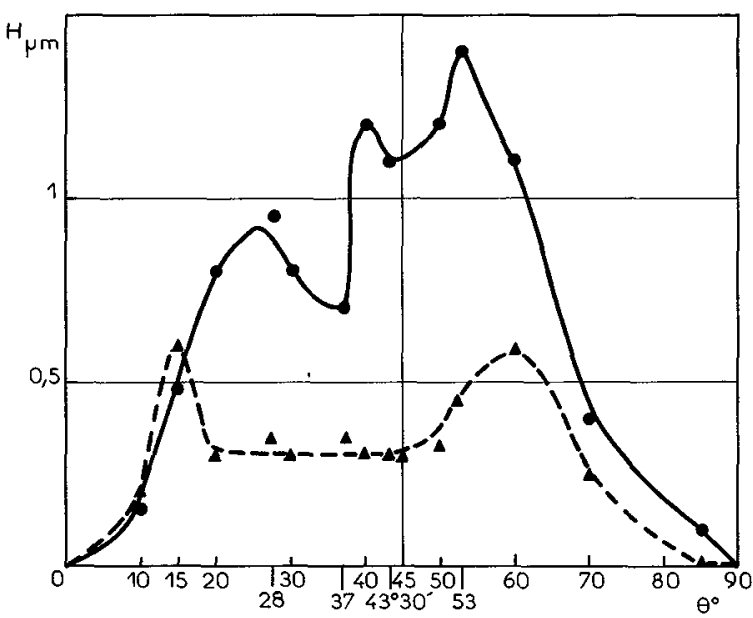

FIG. 6. - Influence de la désorientation $\theta$ sur la profondeur $H$ des sillons d'attaque après maintien de 290 heures dans $\mathrm{HCl} 10 \%$ de bicristaux d'aluminium.

$\longrightarrow$ attaque perpendiculaire à l'axe de flexion $\langle 100\rangle$ $t--\Delta-t$ attaque parallèle à l'axe de flexion $\langle 100\rangle$

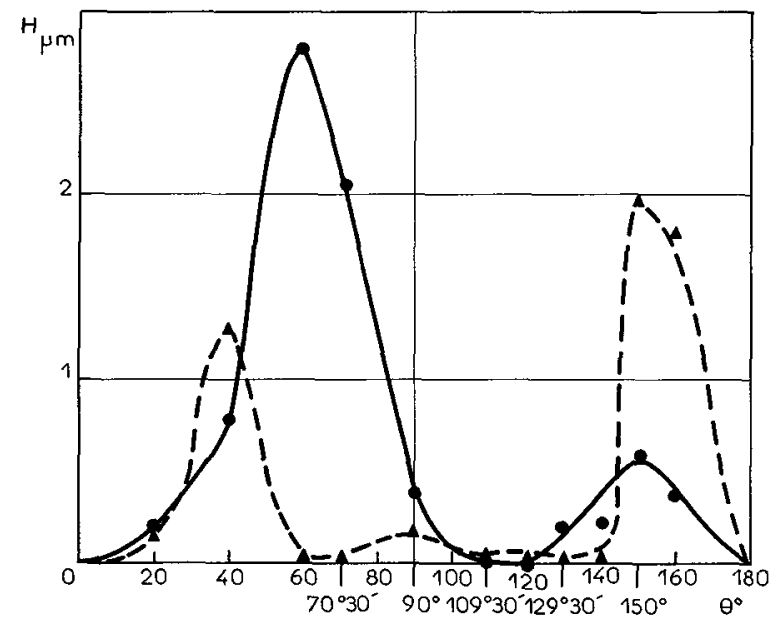

Fici. 7 - Influence de la désorientation $\theta$ sus la profondeur $H$ des sillons d'attaque après maintien de 400 heures dans $\mathrm{HCl} 10 \%$ de bicristaux d'aluminium.

attaque perpendiculaire à l'axe de flexion $\langle 110\rangle$

- - - attaque parallèle à l'axe de flexion $\langle 110\rangle$

élevée. Par ailleurs, la macle $\{211\}-109^{\circ} 30^{\prime}$ qui présente une très bonne résistance à la corrosion ne se distingue pas des joints quelconques au point de vue énergie. La considération de l'énergie intergranulaire est donc tout à fait insuffisante pour expliquer l'importance de la corrosion des joints de grains. Ceci n'est pas très étonnant si l'on remarque que la corrosion intergranulaire est une grandeur vectorielle, sensible à la direction de sa propagation, alors que l'énergie intergranulaire est une grandeur scalaire.

4.2 Structure des JOINTS DE GRains. - Une meilleure compréhension des phénomènes de corrosion intergranulaire doit donc être recherchée dans une corrélation plus étroite avec la structure des joints de grains [17, 41, 39]. Une telle corrélation est plus aisée dans le cas de la corrosion d'un métal comme l'aluminium pour lequel on dispose de données assez nombreuses sur son comportement vis-à-vis de la corrosion $[15,17,41]$ et de modèles de joints de grains [48].

Dans le cas de l'axe de flexion $\langle 100\rangle$, la structure des joints peut être décrite par des défauts linéaires, parallèles à l'axe de flexion. Aux faibles désorientations, le joint est une paroi de dislocations dont la distance mutuelle décroît lorsque la désorientation augmente. Vers $\theta=20^{\circ}$, il se forme des canaux séparés par des zones de cristal où la position des atomes n'est pas perturbée. Ces zones diminuent lorsque la désorientation augmente, pour disparaître à $\theta=53^{\circ}$; le joint est alors une succession ininterrompue de défauts. Au-delà, les zones de bon accord réapparaissent et vers $\theta=70^{\circ}$ on retrouve des dislocations dont le vecteur de Burgers est différent de celui relatif aux dislocations des joints de désorientation voisine de $0^{\circ}$. Ainsi, la dissymétrie des courbes représentant la corrosion de l'aluminium en solution d'acide chlorhydrique en fonction de la désorientation, par rapport à la valeur $\theta=45^{\circ}$, pourrait être rattachée à ces différences d'organisation du joint entre 0-20 et $70-90^{\circ}$. Par ailleurs, on pourrait expliquer le maximum de corrosion, observé au cours de l'attaque perpendiculaire à l'axe de flexion (Fig. 6) pour une désorientation voisine de $50^{\circ}$. Enfin, si l'on considère l'attaque parallèle à l'axe de flexion, le passage d'une organisation en parois de dislocations à des zones de bon accord cristallographique séparées par des canaux pourrait être à l'origine du maximum d'attaque observé pour des désorientations proches de $15^{\circ}$ et $60^{\circ}$. Cependant, il est très difficile de comprendre pourquoi le maximum de corrosion intergranulaire est plus élevé lorsque l'attaque est effectuée perpendiculairement à l'axe.

Dans le cas de l'axe de flexion $\langle 110\rangle$, la considération des structures de joints ne permet pas de rendre compte de la faible réactivité de la macle $\{211\}$ ni du faible taux d'attaque observé pour des désorientations proches de $100^{\circ}$.

Il est certain que le mécanisme même du processus de corrosion doit intervenir et pourrait expliquer s'il était mieux connu certaines des contradictions qui viennent d'être relevées. Ainsi, lors de la corrosion de l'aluminium dans l'eau pressurisée, on attribue un rôle essentiel à la réaction cathodique de formation d'hydrogène moléculaire ; ce dernier peut diffuser plus facilement dans les canaux séparant les deux réseaux; dans ces conditions on observe bien une corrosion plus importante de l'aluminium dans la direction parallèle à l'axe de flexion [49].

Enfin, il faut constater que les problèmes de corrélations entre structure des joints et corrosion intergranulaire déjà complexes avec les métaux deviennent encore plus délicats dans le cas des 
alliages où les positions des atomes des différents constituants au niveau des joints ne sont pas encore connues.

4.2.1 Influence de l'asymétrie. - L L'influence de la structure du joint de grains sur son comportement vis-à-vis de la corrosion se manifeste d'une façon particulièrement nette lorsque le paramètre d'asymétrie du joint intervient. Ainsi, avec les aciers inoxydables, le joint est rarement rectiligne sur toute l'épaisseur du bicristal et il est fréquent de rencontrer toutes les valeurs possibles du paramètre d'asymétrie $\varphi$ entre 0 et $45^{\circ}$. Lorsque l'attaque est perpendiculaire à l'axe de flexion, l'asymétrie se traduit seulement par la formation d'un sillon dissymétrique [43] sans que l'importance de l'attaque (angle d'ouverture $\alpha$ du sillon, profondeur $H$ ) en soit très affectée. Par contre, lorsque l'attaque se propage parallèlement à l'axe de flexion, le paramètre d'asymétrie modifie considérablement les caractéristiques du sillon intergranulaire. La figure 8 montre l'évolution de la largeur $L$ du sillon en fonction de la désorientation $\theta$ et du paramètre d'asymétrie $\varphi$. Pour une désorientation $\theta$, la corro-

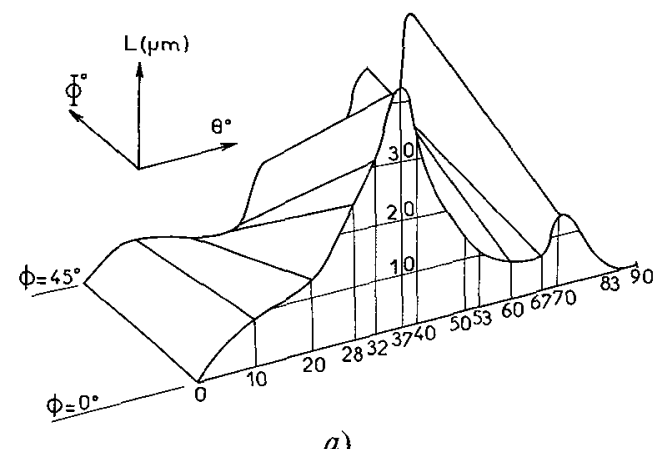

a)

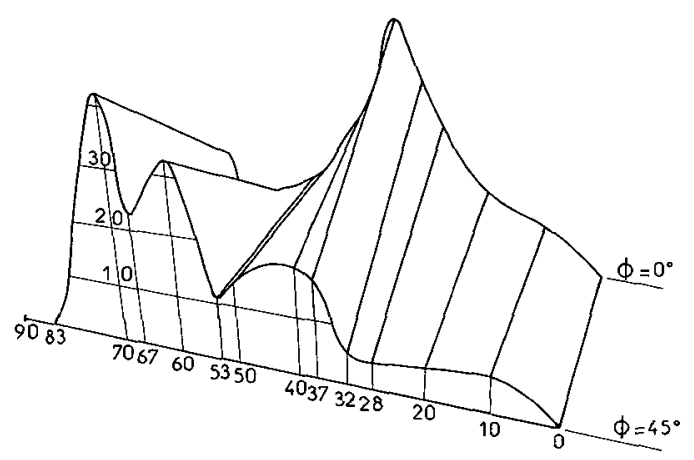

b)

FIG. 8. - Evolution de la largeur $L$ des sillons d'attaque en fonction de la désorientation $\theta$ et du paramètre d'asymétrie $\varphi$; bicristaux d'acier inoxydable d'axe de flexion $\langle 100\rangle$; attaque parallèle à l'axe de flexion dans $\mathrm{H}_{2} \mathrm{SO}_{4} 2 \mathrm{~N}, V=1,1$ V/E.C.S.

sion varie quasi linéairement entre 0 et $45^{\circ}$ d'asymétrie. La pente de ces droites dépend de la désorientation [13]. On peut expliquer en particulier l'inversion du signe de la pente vers $50^{\circ}$ de désorientation en considérant (Fig. 9) pour chaque bicristal le joint symétrique $\theta$ et son complé-

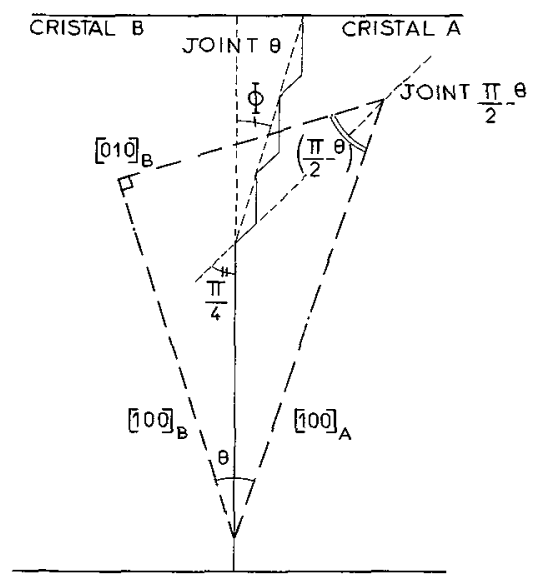

FIG. 9. - Constitution d'un joint de paramètre d'asymétrie $\varphi$ à partir de 2 joints symétriques de désorientation $\theta$ et $\pi / 2-\theta$.

mentaire $\pi / 2-\theta$. On admet que le joint asymétrique est formé de marches révélant des facettes de plus bas indices; ceci serait lié à la plus grande stabilité, dans certains cas, du joint asymétrique et a déjà été observé avec plusieurs métaux $[50,51$, 52]. Comme le montre la figure 9 , le joint asymétri que est formé de marches parallèles soit au joinı symétrique $\theta$, soit au joint $\pi / 2-\theta$. En tenant compte des taux de corrosion des deux joints symétriques, on peut calculer la pente des droites représentant l'évolution de $L$ et $H$ en fonction de $\varphi$. Pour cela, on pondère les taux de corrosion suivant la constitution du joint asymétrique considéré [13].

\section{TABLEAU I}

$\begin{array}{ccc} & \begin{array}{c}\text { Pente } \\ \text { expérimentale. }\end{array} & \begin{array}{c}\text { Pente } \\ \text { calculée }\end{array} \\ \begin{array}{c}\theta=37^{\circ} \\ L\end{array} & - & - \\ H & -0,58 & -0,65 \\ \theta=53^{\circ} & -0,27 & -0,29 \\ L & & \\ H & 0,15 & 0,13 \\ \theta=40^{\circ} & 0,02 & 0,02 \\ L & & -0,32 \\ H & -0,36 & -0,04 \\ \theta=50^{\circ} & -0,05 & 0,11 \\ L & & 0,03 \\ H & 0 & \end{array}$

Le tableau I montre qu'il y a un bon accord entre les valeurs calculées et trouvées expérimentalement dans le cas des couples de joints complémentaires $37-53^{\circ}$ et $40-50^{\circ}$. Ne disposant que d'un nombre limité de bicristaux, il est difficile d'étendre ce genre de calculs à toutes les désorientations.

Cet exemple montre donc que l'étude de la corrosion intergranulaire, accessible d'une façon 
quantitative grâce à des mesures locales tout le long du joint de grains, permet d'apporter quelques données sur la structure de ces joints. Ceci est un avantage vis-à-vis d'autres propriétés des joints de grains comme par exemple la diffusion, pour lesquelles il n'est possible d'atteindre que des valeurs moyennes.

4.3 INTERVENTION DES FACTEURS PROPRES A LA MATRICE. - Jusqu'ici, nous avons cherché à expliquer le phénomène de corrosion intergranulaire en ne considérant que certaines propriétés intrinsèques des joints de grains. En fait, la corrosion intergranulaire ne se localise pas seulement dans les zones où la position des atomes est perturbée par la présence du joint de grains. Nous avons vu, en effet, que les dimensions des sillons intergranulaires atteignent très rapidement des valeurs de l'ordre du micron. Ceci laisse supposer que les caractéristiques de dissolution de la matrice doivent intervenir. La corrosion intergranulaire, telle que nous pouvons l'observer, correspond finalement à une compétition entre une attaque plus rapide dans le plan du joint et la corrosion générale de la matrice. Or, il est bien évident que de nombreux facteurs sont susceptibles de modifier cette corrosion générale. Les conditions d'attaque (milieu agressif, potentiel d'électrode...) jouent un rôle important; mais dans des conditions données d'attaque, il faut tenir compte également des caractéristiques des matériaux. C'est sur ces dernières que nous insisterons plus particulièrement. Nous examinerons ainsi le rôle joué par les phénomènes d'anisotropie de dissolution anodique, en fonction de l'orientation cristalline, ainsi que par la composition des matériaux. Ce dernier point est particulièrement vaste et complexe à aborder car il y a évidemment des relations étroites, et pas toujours très bien connues, entre la composition du matériau, la présence d'impuretés et les propriétés mêmes des joints de grains, modifiées par des phénomènes de ségrégation.

4.3.1 Influence de l'anisotropie de dissolution. - On sait que la vitesse de corrosion dépend souvent de l'orientation cristalline de la surface mise en présence du milieu agressif. Ceci peut jouer sur la corrosion intergranulaire dans la mesure où sur un échantillon polycristallin, l'orientation de surface varie d'un grain à l'autre. Ce phénomène est plus commodément étudié avec des bicristaux attaqués dans une direction perpendiculaire à l'axe de flexion. En effet, au fur et à mesure que la désorientation augmente, les indices de la surface en contact avec l'électrolyte se modifient.

L'aluminium est un cas particulièrement intéressant car en présence d'acide chlorhydrique il présente une forte anisotropie de dissolution [15, 53]. Les mesures effectuées sur des monocristaux ou des bicristaux d'aluminium de même pureté montrent que la face $\{111\}$ se dissout avec une vitesse de $20 \%$ supérieure à celle de la face $\{110\}$ et de $50 \%$ supérieure à celle de la face $\{100\}$ [42]. On peut donc essayer de reprendre les données de corrosion intergranulaire de l'aluminium à la lumière de ces résultats. La figure 10 présente l'attaque intergranulaire de bicristaux d'axe de flexion $<110>$ en considérant cette fois l'angle d'ouverture $\alpha$ du sillon intergranulaire porté en fonction de la désorientation $\theta$. Nous avons également porté sur cette figure des droites représentant l'angle formé par un certain nombre de plans denses peu inclinés par rapport à la surface en cours de dissolution et donc susceptibles d'être dégagés au cours de la corrosion. Cette figure montre que pratiquement tous les points expérimentaux se placent sur une de ces droites. Le fait le plus intéressant à souligner est que pour les désorientations comprises entre 60 et $120^{\circ}$ l'angle $\alpha$ est très proche de plans $\{111\}$ dont on sait que la vitesse de dissolution est rapide. Or, dans le domaine de désorientation autour de $100^{\circ}$, nous avions précédemment constaté une attaque intergranulaire faible, en contradiction avec une énergie des joints élevée. Il est donc très probable que dans ce domaine de désorientation où la surface en cours de dissolution est proche de plans $\{111\}$ attaqués rapidement, l'apparition de sillons profonds n'est pas possible.

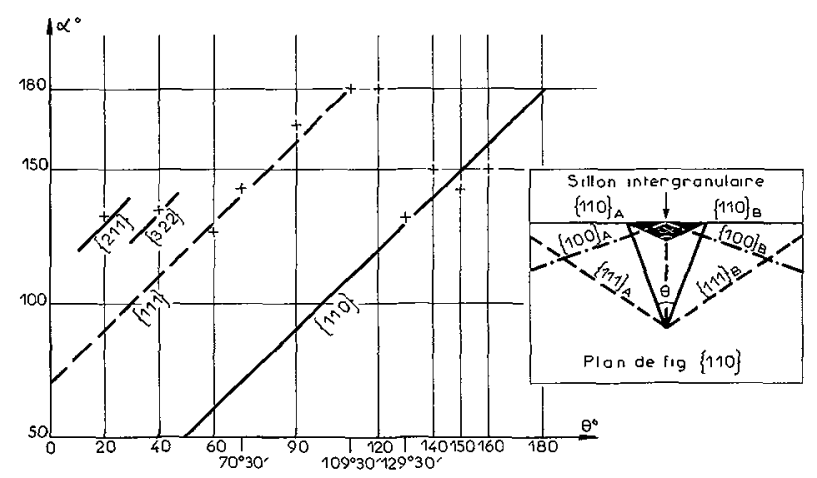

FIG. 10. - Influence de la désorientation $\theta$ sur l'angle $\alpha$ d'ouverture des sillons intergranulaires formés par attaque perpendiculaire à l'axe de flexion $<110\rangle$ de bicristaux d'aluminium ; comparaison avec les droites représentant l'évolution de l'angle formé par diverses familles de plans cristallographiques.

Certains aciers inoxydables présentent également une anisotropie de dissolution anodique en fonction de l'orientation cristalline. Par exemple, avec un acier à $17 \%$ de chrome et $13 \%$ de nickel qui a servi à préparer des bicristaux, on trouve que l'attaque à $1,1 \mathrm{~V} / \mathrm{E}$.C.S. dans l'acide sulfurique $2 \mathrm{~N}$, entraîne une vitesse de dissolution d'une face $\{100\} 50 \%$ plus grande que celle de faces $\{110\}$ ou $\{111\}$. Si l'on se reporte à la figure 5 illustrant l'évolution de la largeur $L$ du sillon en fonction de la désorientation, on constate une très forte dissymétrie par rapport à la valeur de $\theta=45^{\circ}$. 
En particulier, la corrosion augmente lentement entre 0 et $20^{\circ}$, alors que la variation est très rapide au voisinage de $\theta=90^{\circ}$. Or, aux désorientations proches de $0^{\circ}$, la surface est constituée de plans $\{100\}$ se dissolvant rapidement alors que vers $90^{\circ}$, la surface tend vers des plans $\{110\}$ de vitesse de dissolution lente. Un autre fait illustre l'anisotropie de dissolution. Nous avons constaté que les faces du sillon d'attaque correspondent dans la plupart des cas à des plans d'indices simples du type $\{0 \mathrm{kl}\}:\{011\},\{012\},\{023\},\{034\}$. Ceci n'est pas très étonnant, puisque nous avons trouvé que les plans de type $\{0 \mathrm{kl}\}$ se dissolvent plus lentement que ceux de type $\{100\}$. Par ailleurs, on observe peu de différences entre les vitesses de dissolution des divers plans de type $\{0 \mathrm{kl}\}$. Ceci suggère que les valeurs de $\alpha$ ne sont pas très affectées par l'anisotropie de dissolution et que ces valeurs sont bien caractéristiques des propriétés des joints de grains.

Notons que certains matériaux, par exemple le nickel, ne présentent pas d'anisotropie de vitesse de dissolution notable. En conclusion, l'anisotropie de la vitesse de dissolution en fonction de l'orientation cristalline est un facteur dont il faut tenir compte toutes les fois que l'on cherche à établir des corrélations étroites entre la corrosion intergranulaire et les propriétés des joints de grains.

4.3.2 Influence de la composition; phénomènes de ségrégation. - 4.3.2.1 Données générales. Dès les premiers travaux, il est apparu que la composition des matériaux métalliques avait une influence considérable sur leur comportement vis-àvis de la corrosion intergranulaire. Ce problème est d'autant plus complexe à aborder qu'il concerne non seulement des variations de composition liées aux éléments majeurs d'un alliage, mais encore le rôle que peuvent jouer des éléments sous forme d'impuretés à des concentrations extrêmement faibles. Avant même de considérer l'influence de la composition sur certaines propriétés des joints de grains, il ne faut pas perdre de vue que la corrosion générale d'un matériau métallique est également très sensible à sa composition. Or, comme nous venons de le rappeler, la corrosion intergranulaire résulte d'une compétition entre les vitesses d'attaque dans le plan du joint et au niveau de la matrice. Les relations entre composition et corrosion générale ont fait l'objet de nombreux travaux et certains très complets concernent les alliages fer-chromenickel. Ainsi, a été envisagée l'influence des éléments majeurs [54] et des impuretés [54, 55]. Dans ce dernier cas, des formules ont même été établies reliant l'évolution du courant de dissolution et la teneur en divers éléments mineurs [56, 57]. Dans le cas de l'aluminium, mis en contact avec des solutions acides, le rôle des impuretés sur la corrosion générale est considérable [15] [58, 42]. Ainsi, la vitesse de corrosion générale dans l'acide chlorhydrique à $10 \%$ maintenue à $25^{\circ} \mathrm{C}$, passe de $10 \mathrm{mg} \mathrm{cm}^{-2} \mathrm{~h}^{-1}$ pour un aluminium de pureté $99,9 \%$ à $6 \times 10^{-2} \mathrm{mg} \mathrm{cm}^{-2} \mathrm{~h}^{-1}$ pour un aluminium de pureté $99,998 \%$ et $10^{-2} \mathrm{mg} \mathrm{cm}^{-2} \mathrm{~h}^{-1}$ pour de l'aluminium de zone fondue. Or, comme nous l'avon's vu, une augmentation de la vitesse de corrosion générale peut entraîner une diminution de l'attaque intergranulaire.

En ce qui concerne l'influence de la composition sur la corrosion intergranulaire, ce sont les aciers à base de nickel et de chrome qui, compte tenu de leurs applications, fournissent les données les plus nombreuses. Leur analyse est souvent difficile car la plupart des résultats ont été obtenus après maintien de l'acier dans diverses solutions proches de celles utilisées pour les tests de corrosion intergranulaire des aciers sensibilisés. Par ailleurs, la corrosion intergranulaire est souvent estimée d'une façon qualitative, ce qui rend hasardeuse toute comparaison, ou à partir du taux global de corrosion et il est alors difficile de dégager ce qui revient à la corrosion intergranulaire.

Le rôle joué par la composition des matériaux dans la corrosion localisée aux joints de grains a souvent été interprété en invoquant des phénomènes de ségrégation. Il s'agit probablement du paramètre dont il est le plus délicat d'apprécier l'importance sur la corrosion intergranulaire, compte tenu du nombre d'éléments susceptibles d'intervenir et de la difficulté d'atteindre leur concentration au niveau du joint de grains. De très nombreux travaux ont été effectués dans ce domaine et nous renvoyons le lecteur à deux articles de mise au point récents concernant à la fois l'aspect théorique des phénomènes de ségrégation et l'analyse des données de la littérature [61, 62]. C'est probablement dans le cas des aciers que les travaux les plus cohérents ont été effectués ces dernières années. Armijo [63] en particulier, a étudié systématiquement l'influence d'un certain nombre d'éléments ( $\mathrm{Mn}, \mathrm{Si}, \mathrm{C}, \mathrm{S}, \mathrm{P}, \mathrm{N}, \mathrm{O})$ sur la corrosion intergranulaire d'aciers à $15 \%$ de chrome et $12 \%$ de nickel, provoquée par un maintien dans une solution bouillante d'acide nitrique contenant des ions $\mathrm{Cr}^{6+}$. Il apparaît que le phosphore et le silicium, en accord avec d'autres travaux [46, 64, 65], sont particulièrement nocifs pour la corrosion intergranulaire. Cependant, les preuves de la ségrégation intergranulaire ne sont apportées que d'une façon indirecte par des mesures de microdureté [62, 66]. La situation a considérablement évolué ces dernières années, par suite de la mise en œuvre de méthodes comme la spectroscopie des électrons Auger sur des fractures intergranulaires. Un des attraits de cette méthode est d'apporter des informations non seulement sur la nature des éléments ségrégés, mais également sur le mécanisme de cette ségrégation qui peut s'effectuer en équilibre ou hors d'équilibre [67]. L'utilisation de la spectroscopie 
des électrons Auger semble d'ailleurs apporter quelques bouleversements dans les conceptions classiques sur l'épaisseur de la zone touchée lors de la ségrégation à l'équilibre [68, 69] .

Joshi et Stein ont, par spectroscopie d'électrons Auger, montré la ségrégation intergranulaire d'éléments comme le soufre, le silicium, l'azote et le phosphore, pour des aciers au chrome-nickel [70]. La présence de ces éléments dans les joints de grains s'accompagne alors d'une attaque intergranulaire après maintien dans une solution nitrique contenant des ions $\mathrm{Cr}^{6+}$, milieu qui correspond à un maintien potentiostatique au début du domaine de transpassivité ( $V \sim 1100 \mathrm{mV} /$ E.C.S.) où la corrosion intergranulaire est particulièrement importante [71]. 'Il semble maintenant incontestable que ces résultats sont très prometteurs et qu'il s'agit d'une voie d'avenir. Une meilleure compréhension des relations entre ségrégation et corrosion intergranulaire passe en effet par une connaissance approfondie de la nature des éléments ségrégés, de leur concentration et de leur mode de liaison au niveau des joints de grains.

\subsubsection{Corrosion intergranulaire du nickel et} des alliages à base de nickel et de chrome. - La confrontation des données électrochimiques avec des estimations quantitatives de la corrosion intergranulaire peut apporter un certain nombre d'éléments sur le rôle joué par la composition des matériaux et les phénomènes de ségrégation. Nous traiterons plus particulièrement du cas du nickel, d'aciers à base de nickel et de chrome puis de l'influence d'additions de carbone ou de silicium. Ces matériaux présentent en effet une corrosion intergranulaire très marquée lorsqu'ils sont maintenus à des tensions correspondant au début du domaine de transpassivité. La corrosion intergranulaire s'accompagne de la formation de sillons réguliers (Fig. 1 et 2 ) dont le profil d'équilibre est atteint pour de faibles quantités d'électricité ; $2 \mathrm{~cm}^{-2}$ par exemple, dans le cas du nickel. Comme il s'agit de matériaux polycristallins, les diverses désorientations entre les grains entraînent évidemment une certaine dispersion des mesures de l'angle d'ouverture $\alpha$; on se référera donc à des valeurs moyennes correspondant en général à au moins une dizaine de déterminations. La figure 11 est relative au rôle joué par le silicium sur la corrosión intergranulaire du nickel. En attaquant des échantillons de nickel contenant $0,003,1,2,3$ et $4 \%$ de silicium, on obtient les valeurs de $\alpha$ reportées sur la courbe 1 .

L'attaque intergranulaire est la plus intense pour une teneur en silicium de $1 \%$. Il apparaît également qu'aux teneurs élevées en silicium, l'attaque intergranulaire est plus faible que celle observée avec le nickel. Nous expliquons cette évolution en admettant d'abord que la corrosion intergranulaire est le
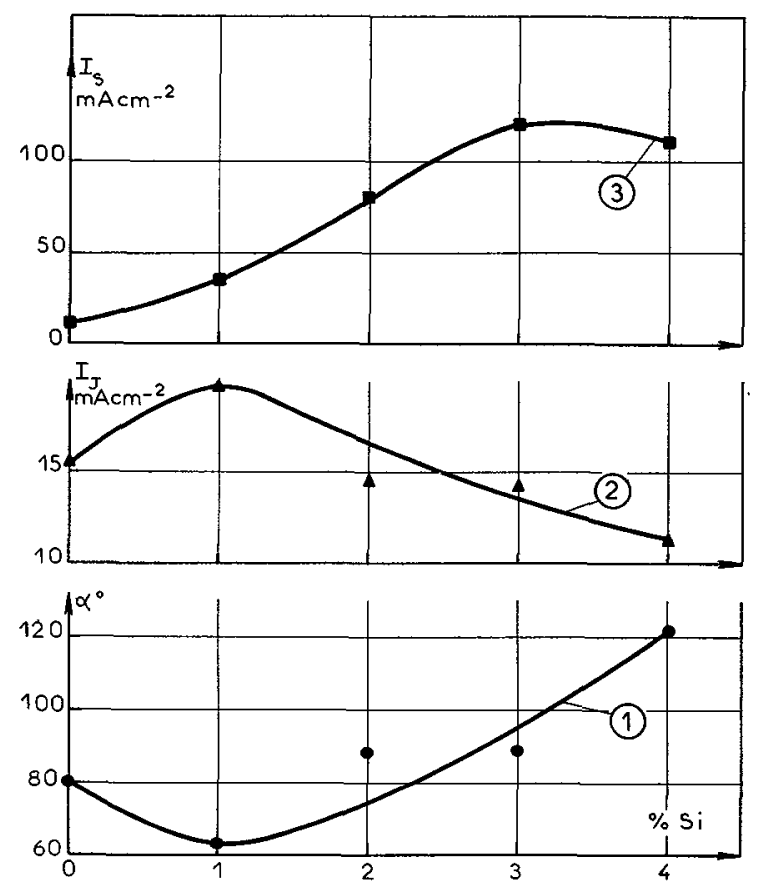

FIG. 11. - Influence de la teneur en silicium sur l'attaque intergranulaire du nickel polycristallin dans $\mathrm{H}_{2} \mathrm{SO}_{4}, 2 \mathrm{~N}$.

Courbe 1: Evolution de l'angle d'ouverture $\alpha$ des sillons d'attaque.

Courbe 2 : Evolution de la densité de courant $I_{\mathrm{I}}$ calculée à partir de l'angle $\alpha$.

Courbe 3: Evolution de la densité de courant de dissolution générale $J_{S}$ mesurée à une tension de 1,3 V/E.C.S.

résultat d'une compétition entre les vitesses de dissolution au niveau des joints et de la matrice et que, par ailleurs, le silicium ségrège au niveau des joints [45]. Le sillon d'attaque ayant atteint un profil d'équilibre, le courant de dissolution au niveau des joints $I_{\mathrm{J}}$ et de la matrice $I_{\mathrm{S}}$ sont donc reliés, comme le montre le schéma de la figure 12 , à l'angle $\alpha$ :

$$
\frac{I_{\mathrm{J}}}{I_{\mathrm{S}}}=\frac{1}{\sin \alpha / 2}
$$

La courbe 2 de la figure 11 montre la variation du courant $I_{\mathrm{J}}$ en fonction de la teneur en silicium. L'influence du silicium sur le courant de dissolution anodique appuie l'hypothèse d'une ségrégation de cet élément au niveau des joints. La courbe 3 de la

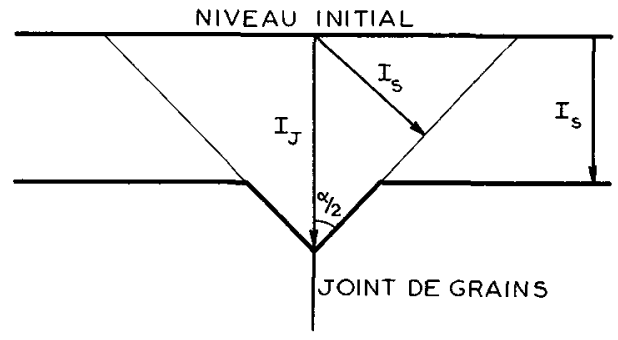

FIG. 12. - Schéma de la formation d'un sillon d'attaque intergranulaire à partir des courants de dissolution de la matrice $I_{S}$ et des zones intergranulaires $I_{\mathrm{J}}$. 
figure 11 donne, à titre d'exemple, les valeurs du courant de dissolution anodique $I_{S}$ repérées sur des échantillons maintenus à une tension de 1,3 V/E.C.S. On voit que le silicium provoque d'abord une forte augmentation de $I_{S}$, mais qu'audelà de $3 \%$ en silicium, le courant tend à diminuer. Si le silicium ségrège au niveau des joints, on comprend qu'aux faibles teneurs de la matrice corresponde un courant $I_{\mathrm{J}}$ élevé et donc une forte corrosion intergranulaire. Par contre, au-delà d'une certaine teneur, ici $1 \%$, l'enrichissement en silicium au niveau du joint serait tel que le courant $I_{\mathrm{J}}$ tendrait à diminuer. Comme c'est finalement le rapport $I_{\mathrm{J}} / I_{\mathrm{S}}$ qui impose la géométrie du sillon, une diminution relative de $I_{S}$ par rapport à $I_{S}$ entraînerait une corrosion intergranulaire de l'alliage à $4 \%$ de silicium inférieure à celle observée avec le nickel.

La mesure de l'angle d'ouverture $\alpha$ permet également d'apprécier l'influence de la composition dans le cas des aciers à base de chrome et de nickel. Les tableaux II, III, IV, V regroupent un certain nombre de résultats dont certains ont été obtenus récemment par L. Beaunier et C. Vignaud ; ils sont relatifs à des attaques effectuées dans une solution aqueuse d'acide sulfurique $2 \mathrm{~N}$ à des potentiels correspondant au domaine de transpassivité.

\section{TABleau II}

$$
\begin{array}{ccccccc}
\mathrm{Ni} \% & 11,2 & 32,9 & 45,1 & 54,7 & 66,6 & 74,6 \\
\overline{\alpha^{0}} & \overline{145} & \overline{112} & \overline{88} & \overline{78} & \overline{75} & \overline{75}
\end{array}
$$

Le tableau II illustre l'influence du nickel dans le cas d'aciers à $18 \%$ de chrome: la teneur en carbone est comprise entre 0,002 et $0,005 \%$ et la teneur en silicium ne dépasse pas $0,1 \%$. Comme cela a déjà été signalé [59] l'augmentation de la teneur en nickel intensifie l'attaque intergranulaire malgré une forte augmentation du courant de dissolution générale; ce dernier passe de 1,9 à $13 \mathrm{~mA} \mathrm{~cm}^{-2}$ lorsque la teneur en nickel varie de 11,3 à $74,6 \%$ (tension d'électrode $V=1,1$ V/E.C.S.). L'acier à $75 \%$ de nickel a finalement un comportement très voisin du nickel puisque l'angle $\alpha$ a une valeur voisine de $75^{\circ}$ pour ces 2 matériaux attaqués dans les mêmes conditions.

\section{TABleaU III}

$$
\begin{array}{ccccccc}
\mathrm{Cr} \% & 8 & 10 & 12 & 14 & 16 & 18 \\
\overline{\alpha^{0}} & \overline{120} & \frac{-}{110} & \frac{-}{100} & \overline{60} & \overline{70} & 70
\end{array}
$$

Le tableau III montre que le chrome a un effet analogue à celui du nickel ; les aciers correspondant au tableau III contiennent $77 \%$ de nickel, de 0,002 à $0,003 \%$ de carbone et moins de $0,1 \%$ de silicium. L'augmentation du courant de dissolution générale provoqué par le chrome n'empêche pas une diminution très significative de l'angle $\alpha$. A composition donnée en chrome et nickel la teneur en carbone ou en silicium joue également un rôle très important. L'influence du silicium a été étudiée dans le cas d'aciers à $16 \%$ de chrome et $14 \%$ de nickel (Tableau IV)

Tableau IV

$\begin{array}{cccccc}\text { Si } \% & 0,12 & 0,41 & 0,94 & 1,32 & 1,99 \\ \overline{\alpha^{0}} & \overline{147} & \overline{120} & \overline{105} & \overline{110} & \overline{142}\end{array}$

On note comme pour le nickel un maximum de la corrosion intergranulaire pour une teneur en silicium voisine de $1 \%$.

$$
\begin{array}{cccc}
\multicolumn{4}{c}{\text { TableaU V }} \\
0 \% & 0,007 & \mathbf{0 , 0 1 7} & \mathbf{0 , 0 3 3} \\
\overline{\alpha^{0}} & \overline{75} & \overline{60} & \overline{50}
\end{array}
$$

Le tableau $\mathrm{V}$ est relatif à des aciers contenant $16 \%$ de chrome et $75 \%$ de nickel. L'augmentation de la teneur en carbone entraîne une corrosion intergranulaire légèrement accrue. $\mathrm{Ce}$ résultat confirme des données obtenues avec une série d'aciers à $16 \%$ de chrome et $14 \%$ de nickel dont la teneur en carbone est comprise entre 0,02 et $0,2 \%$ [60]. En outre les observations microscopiques révèlent une forte évolution du faciès de l'attaque ce qui souligne une fois encore le fait que l'on ne peut pas se contenter de données globales sur la corrosion intergranulaire. En présence de teneurs élevées en carbone, les sillons d'attaque ne sont pas réguliers et se présentent sous forme d'alignements de piqûres. Ceci laisse supposer une précipitation locale de carbures. A ces teneurs en carbone, les coefficients de diffusion intergranulaire de ${ }^{59} \mathrm{Fe}$, ${ }^{51} \mathrm{Cr}$ et ${ }^{63} \mathrm{Ni}$, sont d'ailleurs très notablement diminués. Les précipitations de carbures de chrome entraînent probablement une réduction de la longueur totale des joints participant à la diffusion et provoquent l'attaque très irrégulière des zones intergranulaires.

Nous ne devons pas, également, perdre de vue que l'augmentation de la teneur en carbone et en silicium entraîne une forte augmentation de la vitesse de corrosion générale. Corrélativement, on observe une élévation des coefficients d'autodiffusion en volume, liée à une plus grande imperfection du réseau cristallin. La densité importante des défauts ponctuels est alors susceptible de modifier les constantes cinétiques de la réaction de dissolution anodique. L'augmentation de l'attaque intergranulaire, malgré une élévation du niveau de 
corrosion générale, pourrait s'expliquer par une ségrégation de ces éléments au niveau des joints de grains $[45,60]$.

4.3.2.3 Validité des corrélations entre la structure des joints de grains et la corrosion intergranulaire ; étude des matériaux très purs. - Le rôle joué par la composition des matériaux sur la corrosion générale ou localisée et l'intervention de phénomènes de ségrégations obligent à s'interroger sur la validité des corrélations que l'on s'est efforcé d'établir entre la structure intrinsèque des joints de grains et leur comportement vis-à-vis de l'attaque intergranulaire. A priori, il semble difficile de séparer ce qui revient d'une part à l'organisation des atomes au niveau des joints de grains d'un matériau idéalement pur, d'autre part, aux ségrégations d'impuretés inévitables avec les matériaux couramment utilisés. En effet, des joints où le désordre atomique est élevé sont susceptibles d'avoir une plus grande interaction avec les impuretés que des joints à haut degré de coïncidence et par conséquent subir une corrosion accélérée. Une réponse à ces questions réside dans la mise en œuvre de matériaux de plus en plus purs conduisant à se rapprocher du comportement intrinsèque des joints de grains vis-à-vis de leur attaque préférentielle.

Montuelle et ses colloborateurs ont étudié la corrosion intergranulaire d'aciers inoxydables et d'inconels de haute pureté grâce à des maintiens dans des solutions d'acide nitrique contenant des ions $\mathrm{Cr}^{6+}$ [72] ; quelques résultats obtenus récemment sont présentés au cours de ce colloque. Une conclusion importante est que ces matériaux très purs, à l'état hypertrempé, présentent encore une corrosion intergranulaire.

On dispose également de nombreux résultats, parfois contradictoires, dans le cas de l'aluminium. D'après Montariol, la corrosion intergranulaire de l'aluminium de zone fondue serait inférieure à celle de l'aluminium raffiné [73]. Perryman, de son côté, a constaté qu'après trempe depuis $640^{\circ} \mathrm{C}$, l'importance de l'attaque intergranulaire de l'aluminium augmente avec la teneur en fer [74] ; ceci serait lié à une ségrégation à l'équilibre du fer au niveau des joints de grains. Or, Metzger et Arora pensent que le fer ne serait responsable que d'une corrosion localisée sous forme de piqûres [75]. Les données, présentées par Metzger au cours de ce colloque, semblent bien confirmer que ces impuretés n'ont pas d'interaction avec l'attaque intergranulaire de l'aluminium [76].

Les résultats que nous avons obtenus avec l'aluminium confirment ces conclusions. Ainsi, nous avons examiné l'évolution de l'état de surface de l'aluminium, mis en présence d'une solution aqueuse d'acide chlorhydrique, le long des bicristaux ; on sait en effet que le mode préparation des bicristaux entraîne une augmentation de la teneur en impuretés de la tête vers la queue; ceci se traduit par une variation du taux global de corrosion. Ce dernier augmente par exemple de 1,8 à $2,5 \mathrm{mg} \mathrm{cm}^{-2} \mathrm{~h}^{-1}$ lorsqu'on passe de la tête à la queue d'un bicristal d'axe de flexion $<100>$ et de désorientation $53^{\circ}$. Or, on observe que le faciès et les caractéristiques géométriques du sillon d'attaque intergranulaire ne se modifient pas lorsqu'on passe de la tête à la queue du bicristal ; seule la densité de figures de corrosion disposées d'une façon aléatoire augmente régulièrement. Ce fait est confirmé par l'examen en microscopie électronique à balayage de la corrosion intergranulaire d'aluminium de zone fondue (Fig. 13). Le faciès de

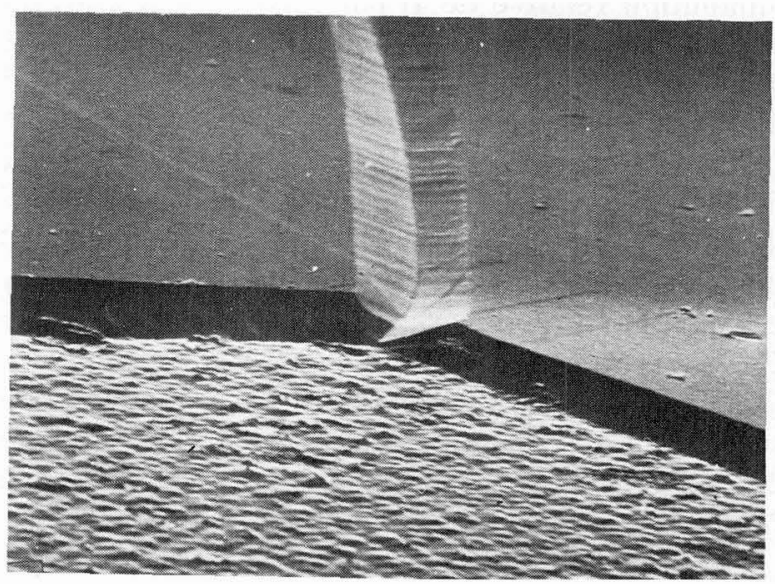

FIG, 13. - Examen en microscopie électronique à balayage d'aluminium de zone fondue après maintien de 290 heures dans $\mathrm{HCl} 10 \%$. $G=1500$.

l'attaque est tout à fait similaire à celui obtenu avec un bicristal de pureté moindre (Fig. 3). Enfin, nous avons cherché à révéler un éventuel enrichissement en impuretés des zones intergranulaires par la formation de figures de corrosion dans des réactifs du type de ceux proposés par Lacombe et Beaujard [77]. En effet, comme l'a montré Wyon, seules les dislocations associées à des atmosphères d'impuretés s'attaquent au contact de ces réactifs [78]. Nous avons constaté que la tête des bicristaux conduit à la formation d'une très faible densité de figures de corrosion. Dans la zone médiane, la répartition des figures de corrosion est pratiquement uniforme et les joints ne sont pas marqués. Des densités de figures d'attaque relativement importantes ne sont observées que sur la queue des bicristaux [17]. Sauf pour quelques rares cas, nous avons constaté que les joints ne sont pas marqués. Pour un certain nombre de bicristaux, correspondant le plus souvent à des joints quelconques, ces derniers sont bordés d'une zone de plusieurs microns, libre des figures d'attaque (Fig. 14). On peut penser que l'interaction des lacunes avec le joint a réduit la possibilité de diffusion des impuretés dans les zones intergranulaires. Les dislocations non décorées ne peuvent donc pas être révélées au cours de l'attaque. Dans 


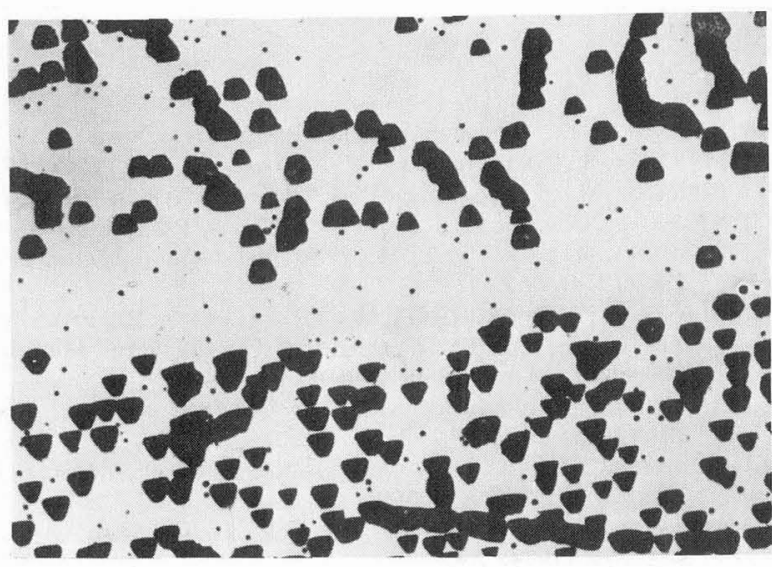

Fig. 14. - Figures de corrosion formées sur la queue d'un bicristal d'aluminium d'axe de flexion $\langle 110\rangle$ et de désorientation $120^{\circ}$ : attaque perpendiculaire à l'axe de flexion dans un mélange de $\mathrm{HCl}, \mathrm{HNO}_{3}, \mathrm{H}_{2} \mathrm{O}$.

un certain nombre de cas, on observe au contraire une distribution uniforme des figures d'attaque (Fig. 15). Ceci est particulièrement net dans le cas des macles $\{111\},\{211\}$ et $\{311\}$. Ceci laisse supposer que l'absence d'interaction avec les lacunes est due à une très bonne organisation des atomes au voisinage de ces joints. Rappelons que la résistance à la corrosion intergranulaire des macles

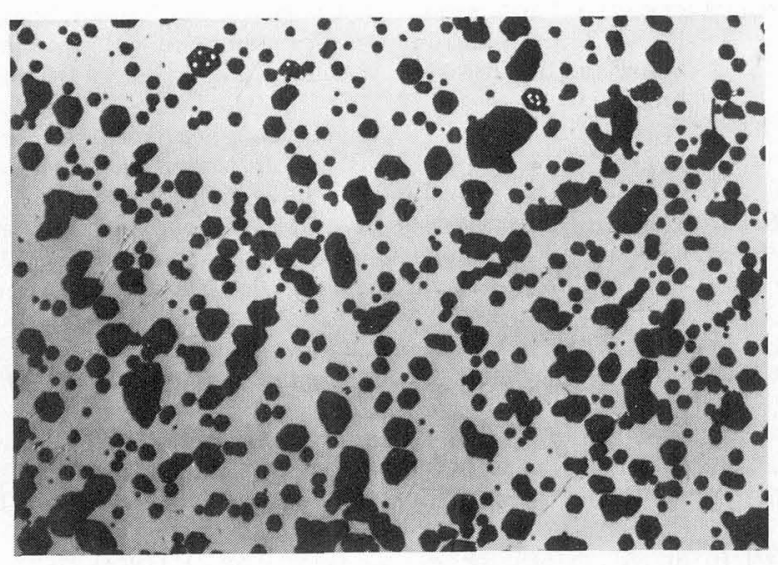

FIG. 15. - Figures de corrosion obtenues dans les mêmes conditions que la figure 14. Bicristal d'aluminium d'axe de flexion $\langle 110\rangle$ et de désorientation $109^{\circ} 30^{\prime}$.

$\{211\}$ et $\{311\}$ a été trouvée excellente (Fig. 7). L'ensemble des résultats que nous venons de présenter dans le cas de l'aluminium semble donc confirmer que les impuretés ne jouent pas un rôle fondamental dans la corrosion intergranulaire. Cette conclusion conforte les relations établies auparavant entre les propriétés intrinsèques des joints de grains et la corrosion intergranulaire.
5. Conclusion. - L'attaque préférentielle des zones intergranulaires, même en absence de précipitations, est apparue ces dernières années comme un phénomène touchant de très nombreux métaux et alliages travaillant dans des conditions extrêmes de pression, température et à des potentiels d'oxydoréduction élevés. Cette corrosion localisée peut entraîner une désagrégation rapide des matériaux ; elle a donc suscité d'assez nombreux travaux depuis une quinzaine d'années. Cependant, la corrosion intergranulaire est un phénomène complexe mettant en jeu de nombreux paramètres liés aux conditions électrochimiques de l'attaque, aux propriétés intrinsèques des joints de grains et des matériaux.

La mise en cuvre de bicristaux permettant de disposer de joints de grains aux caractéristiques cristallographiques bien déterminées et les progrès récents effectués par certaines méthodes de caractérisation des surfaces, ont conduit à dégager le rôle des paramètres essentiels. L'accès à des données quantitatives sur la corrosion intergranulaire a permis d'apporter des éléments d'appréciation sur la validité des modèles représentant la structure des joints de grains.

Nous avons montré que l'établissement d'un sillon d'attaque ayant atteint un profil d'équilibre résulte de la compétition entre les vitesses de dissolution au niveau de la matrice et des zones intergranulaires. La vitesse de dissolution des zones intergranulaires est évidemment rattachée très étroitement aux propriétés intrinsèques des joints de grains et il est certain qu'une meilleure compréhension de la corrosion intergranulaire est liée à l'existence de données précises sur l'arrangement des atomes au niveau des joints de grains. Nous avons vu quelles perturbations apportait la ségrégation de certains éléments au niveau des joints de grains. La mise en œuvre de matériaux de très haute pureté et le développement des méthodes d'analyse des zones intergranulaires devraient conduire à une meilleure appréciation du rôle joué par les phénomènes de ségrégation.

Nous avons souligné qu'il ne fallait pas négliger la vitesse de dissolution de la matrice qui dépend essentiellement de sa composition et des conditions électrochimiques de l'attaque. Cependant le rôle joué par certains éléments, même à faible teneur, et par les défauts ponctuels, dans la vitesse de dissolution des matériaux métalliques ou dans les propriétés des couches passives n'est pas encore très clair. Les efforts poursuivis actuellement pour mieux connaître le mécanisme de la dissolution anodique et de la passivation contribueront certainement à éclaircir les phénomènes d'initiation et de propagation de la corrosion intergranulaire. 


\section{Bibliographie}

[1] LIFKa, B. W., SprowlS, D. D., A.S.T.M. Spec. Tech. Publ. U.S.A., $n^{\circ} 516$ (1972) 120.

[2] Wilson, F. G., Pickering, F. B., J. Iron Steel Inst. 210 (1972) 37.

[3] Henthorne, M., A.S.T.M. Spec. Tech. Publ., U.S.A. $\mathrm{n}^{\circ} 516(1972) 66$.

[4] L'hydrogène dans les métaux, Congrès International, Châtenay-Malabry, Mai 1972.

[5] La corrosion sous tension et la fragilisation par l'hydrogène des alliages à base de fer, Colloque International, Firminy, Juin 1973

[6] Lacombe, P., Yannaquis, N., C.R. Hebd. Séan. Acad. Sci. 224 (1947) 921.

[7] Lacombe, P., Yannaquis, N., Rev. Metall. 45 (1948) 68.

[8] Streicher, M. A., J. Electrochem. Soc. 106 (1959) 161.

[9] Epelboin, I., Froment, M., Morel, P., Corros. Anticorros. 8 (1960) 383

[10] Coriou, H., Hure, J., Plante, G., Electrochim. Acta 5 (1961) 105.

[11] Buhler, H. E., Werkst. Korros. 8 (1966) 661.

[12] Leggert, J. R., PaXton, H. W., Corros. Sci. 2 (1962) 217.

[13] Assassa, W., Beaunier, L., Desestret, A., Froment, M., GuiraldenQ, P., Scr. Metall. 8 (1974) 533.

[14] Monnier, A., Pinard-Legry, G., Comm. au $5^{\text {e }}$ Congrès Européen de Corrosion, Paris 1973, Résumés détaillés p. 245.

[15] ARORA, O. P., Metzger, M., Trans, T.M.S. AIME 236 (1966) 1205.

[16] Hasson, G., Boos, J. Y., Herbeuval, I., Biscondi, M., Goux, C., Surf. Sci. 31 (1972) 115

[17] Froment, M., Vignaud, C., Métaux Corrosion Industries $n^{\circ} 581$ (1974) 1 et $n^{\circ} 582$ (1974) 67.

[18] Montariol, F., Corros. Anticorros. 6 (1958) 101.

[19] Hendrickson, L. E., Metzger, M., Trans. AIME, 242 (1968) 2329

[20] Montuelle, J., Corrosion Protection Finition, 16 (1968), 279.

[21] Gabrielli, C., Métaux Corrosion Industries, 573 (1973) 171, 574 (1973) 223, 577 (1973) 309, 578 (1973) 356.

[22] Froment, M., Vignaud, C., C.R. Hebd. Séan. Acad Sci. 272 (1971) 165.

[23] Froment, M., Conférence aux Journées du SITS-Paris (1971) 47.

[24] Zahavi, J., Metzger, M., J. Electrochem. Soc. (1972) 1479.

[25] Scamans, G. M., Swann, P. R., Communication au Colloque International « La corrosion sous tension et la fragilisation par l'hydrogène des alliages à base de fer ». Firminy, Juin 1973.

[26] Epelboin, I., Froment, M., Garreau, M., Aida, H., $J$. Microsc. 15 (1972) 313.

[27] Flis, J. Br., Corros. Int. 3 (1968) 182.

[28] Garreau, M., Métaux Corrosion Industrie, n 541 (1970) 3 et $n^{\circ} 544(1970) 1$.

[29] ARmiJo, J. S., Wilde, B. E., Corros. Sci. 8 (1968) 649.

[30] Kaischew, Budevski, E., Contemp. Phys. 8 (1967) 489.

[31] Schaarwächter, W., Phys. Stat. sol. 12 (1965) 375 et 865.

[32] Nanev, K. M., KaISCHEW, R., Bulg. Akad Nauk Izvt. Otdel Khim. Nauk. 5 (1972) 19.

[33] Engell, H. J., Steen, A., Rothendacher, P., Communication au $5^{\mathrm{e}}$ Colloque International sur la Corrosion Métallique, Tokyo, 1972, résumés p. 13.

[34] Daguenet, M., Froment, M., Keddam, M., J. Microsc. 5 (1966) 569.

[35] Desestret, A., Epelboin, I., Froment, M., GuiraldenQ, P., Corros. Sci. 8 (1968) 225.

[36] Blondead, G., Froelicher, M., Froment, M., Hugot-Le GofF, A., Phys. Stat. Sol. A, 26 (1974) 181.

[37] SchatT, W., WoRCh, M., Corros. Sci. 9 (1969) 869.

[38] TEDMON, C. S., VERMILYEA, D. A., ROSOlOWSKI, J. H., $J$. Electrochem. Soc., 118 (1972) 192.
[39] Beaunier, L., Thèse $3^{\text {e }}$ Cycle, Paris, Décembre 1973.

[40] Mieluch, J., Smialowski, M., Corros. Sci. 4 (1964) 237.

[41] Boos, J. Y., Thèse Paris 1971, A.O. C.N.R.S. no 5807.

[42] Vignaud, C., Thèse d'Université, Paris 1973.

[43] Beaunier, L., Froment, M., C.R. Hebd. Séan. Acad. Sci. 278 (1974) 667

[44] Desestret, A., Froment, M., Guiraldene, P., Proceedings of the fourth International Congress on Metallic Corrosion, Amsterdam (1969), p. 448.

[45] Beaunier, L., Froment, M., C.R. Hebd. Séan. Acad. Sci. 279 (1974) 91.

[46] Desestret, A., Froment, M., Guiraldene, P., Mém. Sci. Rev. Met. 66 (1969) 389.

[47] Assassa, W., GuiraldenQ, P., C.R. Hebd. Séan. Acad. Sci. 279 (1974) 59

[48] Hasson, G., Thèse Paris 1972, A.O. C.N.R.S. no 6818

[49] Boos, J. Y., Goux, C., Communication au Congrès International "L'hydrogène dans les métaux », Châtenay-Malabry, 1 (1972) 133.

[50] Bishop, G. M., Hart, W. H., Bruggeman, G. A., Acta Metall. 19 (1971) 37.

[51] Weins, M. J., Weins, J. J., Phil. Mag. 26 (1972) 885.

[52] Morgan, A., RalPh, B., Acta Metall. 15 (1967) 341.

[53] Bussy, P., Publ. Sc. Tech. Min. Air no 325 (1957).

[54] Desestret, A., Thèse Paris, 1964, Editions du Cercle d'Etudes des Métaux de St-Etienne.

[55] Streicher, M. A., Corros. 29 (1973) 337.

[56] Wilde, B. E., Greene, N. D., Corros. 25 (1969) 300.

[57] Troselius, L., Corros. Sci. 11 (1971) 473.

[58] Chaudron, G., Monographies sur les métaux de haute pureté, rédigé par Rével G., (Masson), 1972, T. 1, p. 458 .

[59] Coriou, H., Grall, L., Mahieu, C., Pelras, M., Corros. 22 (1966) 280.

[60] Assassa, W., Beaunier, L., Froment, M., Guiraldene, P., Vignaud, C., Communication au $6^{\circ}$ congrès International sur la Corrosion Métallurgique, Sydney, Décembre 1975 .

[61] Aust, K. T., IWAO, D., Communication à la Conférence Internationale U.R. Evans, sur la Corrosion localisée, Williamsburg, Décembre 1971, M.A.C.E. (1974) 62.

[62] CoWan, R. L., Gordon, G. M., Communication au Colloque International sur la Corrosion sous tension et la fragilisation par l'hydrogène des alliages à base de fer, Firminy, juin 1973.

[63] ARmiJo, J. S., Corros. Sci. 24 (1968) 24.

[64] Coriou, H., Desestret, A., Grall, L., Hochmann, J., Rev. Métall. 3 (1964) 177.

[65] Montuelle, J., Mem Scient. Rev. Métallurgie, $\mathrm{n}^{\circ}$ Spécial Colloque sur le fer de très haute pureté, (1968) 372.

[66] Aust, K. T., ARmio, J. S., KoCh, E. F., Westbrook, J. H., Trans. ASM, 61 (1968) 270.

[67] Joshi, A., Stejn, D. F., J. Test. Eval. 1 (1973) 202.

[68] Seah, M. P., Hondros, E. D., Scr. Metall. 3 (1973) 735.

[69] SEaH, M. P., Hondros, E. D., Proc. of the Royal Society of London, 335 (1973) 191.

[70] Joshi, A., Stein, D. F., Corros. 28 (1972) 321.

[71] Brown, H., Corros. 29 (1973) 384.

[72] Montuelle, J., Corrosion, Traitements, Protection, 16 (1968) 279.

[73] Montariol, F., Corros. Anticorros. 6 (1958) 101.

174] Perryman, E. C. W., Trans. AIME 197 (1953) 911.

[75] Metzger, M., Arora, O. P., Trans. AIME 227 (1963) 1305.

[76] Merzger, M., Communication au Colloque International C.N.R.S. sur les joints de grains dans les métaux, St-Etienne, Juin 1975

[77] Lacombe, P., Beaujard, L., Rev. Métall. 45 (1948) 317.

[78] Wyon, G., Métaux Corrosion Industrie, no 541 (1970) 308. 


\section{DISCUSSION}

M. BISCONDI : Dans le cas de corrosion des bicristaux d'aluminium, de flexion autour de $<110\rangle$, dans une solution d'acide chlorhydrique, on remarque une corrosion très marquée de la macle (111), parallèlement ou perpendiculairement à l'axe de flexion, je ne m'en souviens plus. Comment - interprétez-vous cè résultat a priori surprenant ?

M. FROMENT : Effectivement la macle (111) est attaquée lorsque la corrosion a lieu perpendiculairement à l'axe $\langle 110\rangle$ mais ne réagit pratiquement pas pour une corrosion parallèle à l'axe. La comparaison du comportement de cette macle avec les macles (211) et (311) est évidemment difficile compte tenu de la très forte anisotropie de dissolution lorsque l'attaque a lieu perpendiculairement à l'axe. Des calculs sur l'énergie de ces macles donnés au cours du colloque par R. C. Pond, semblent indiquer des valeurs du même ordre de grandeur (60 à $\left.150 \mathrm{ergs} / \mathrm{cm}^{2}\right)$. Ceci coïnciderait avec nos résultats concernant l'attaque parallèle à l'axe $\langle 110\rangle$ (où les effets d'anisotropie de dissolution n'interviennent pas) et qui montrent que les macles (111) (211) et (311) subissent une faible corrosion intergranulaire.

M. BISCONDI : Dans la mesure du possible, il serait préférable de présenter les résultats en reportant non pas une corrosion intergranulaire brute, mais la corrosion intergranulaire vraie, obtenue à partir de la corrosion brute, corrigée par la dissolution des parties monocristallines adjacentes.

J. LEVY : Dans certains cas, notamment au cours du polissage électrolytique, on observe un relief, au lieu d'un sillon, au niveau du joint de grains.

1) Avez-vous fait des observations analogues ?

2) Si oui, quelle est votre interprétation de ce phénomène?

M. Froment : 1) Après de très nombreux polissages électrolytiques de séries de bicristaux d'aluminium ou d'acier inoxydable nous n'avons jamais observé ce phénomène.

2) L'existence de ce phénomène est peut-être liée à des conditions de polissage électrolytiques (concentration des solutions, tension anodique) trop éloignées des conditions optimales. Le contrôle du courant par la diffusion convective n'est plus alors assuré et des irrégularités superficielles peuvent alors se développer.
H. F. MATARE : I am not certain how to consider stress compensation by impurities. As you have shown and as is known, certain impurities will decrease the strain within the grain-boundary. Is there correlation between decrease of strain within the grain-boundary and etch-attack viz. angle $\alpha$ ?

Is E.G. the non attack of certain grainboundaries, you showed explained by this mechanism of stress compensation?

M. FROMENT : Pour répondre correctement à cette question il faudrait associer, aux essais de corrosion décrits dans mon rapport, des expériences de corrosion sous contrainte des mêmes matériaux.

Malgré tout je pense que les effets auxquels vous faites allusion doivent avoir pour conséquence une augmentation de l'énergie du joint.

Dans ces conditions on observe une augmentation de la corrosion intergranulaire (cas des alliages $\mathrm{Fe}-\mathrm{Cr}-\mathrm{Ni}$ ou du $\mathrm{Ni}$ contenant du silicium).

E. D. Hondros : Est-ce que l'angle d'attaque $\alpha$ reste constant pour toute la durée de la corrosion jusqu'à la rupture du métal ?

M. Froment : Une fois passé le stade d'initiation, l'angle d'attaque $\alpha$ reste effectivement constant. Il faut néanmoins souligner qu'une attaque générale irrégulière peut finalement rendre difficile la mesure de cet angle.

G. WYON: A propos de la présence ou de l'absence de zone appauvrie en figures de corrosion de part et d'autre des joints de bicristaux je voudrais signaler que, dans le cas de polycristaux d'aluminium raffiné, le comportement des joints est fortement affecté par le traitement thermique subi par l'échantillon.

Pour un joint donné on constate par exemple une zone appauvrie en figures de corrosion après un refroidissement lent depuis $640^{\circ}$. La largeur du liseré est d'autant plus large que le refroidissement est plus lent.

Par contre pour des vitesses de trempe très élevées, il y a inversion du phénomène et les joints se corrodent préférentiellement. L'état des impuretés de l'aluminium dans les joints, et notamment celui du fer, ainsi que les contraintes introduites par la trempe (plus fortes près des joints) expliquent sans doute ce phénomène. 